\title{
The dynamical transition to step-flow growth during molecular-beam epitaxy of $\mathrm{GaAs}(001)$
}

\author{
M.D. Johnson, J. Sudijono, A.W. Hunt and B.G. Orr \\ The Harrison M. Randall Laboratory, University of Michigan, Ann Arbor, M1 48109-1120, USA
}

Received 17 March 1993; accepted for publication 15 July 1993

\begin{abstract}
Scanning tunneling microscopy studies have been performed on GaAs homoepitaxial films grown by molecular-beam epitaxy. Images show that in the earliest stages of deposition the morphology oscillates between one with two-dimensional islands and flat terraces. After the initial transient regime, the system evolves to a dynamical steady state. This state is characterized by a constant step density and as such the growth mode can be termed step flow. Comparison with RHEED shows that there is a direct correspondence between the surface step density and the RHEED specular intensity. Furthermore, thick films (up to 1450 monolayers) display a constant or slowly increasing surface roughness consistent with long adatom diffusion lengths and limited upward diffusion.
\end{abstract}

For more than a decade the molecular-beam epitaxy (MBE) growth technique has been utilized by both device engineers and scientists to great advantage [1]. It affords monatomic layer thickness control over films growing from the vapor phase at relatively low temperatures under supersaturation conditions. A highly non-equilibrium situation which exists during MBE has enabled the creation of multi-layered structures in which individual layers maintain their chemical integrity and form compositionally abrupt interfaces with one another. This has led to the fabrication of heterostructures and superlattices, which in the case of semiconductor systems, possess novel electrical and optical properties. For optimum performance of devices based on these artificial materials, the morphological sharpness of the interfaces is of primary importance. Roughness leads to increased carrier scattering in active regions, lowering electron and hole mobilities. It also causes variations in the quantum well width which broaden exciton linewidths. As interface smoothness is ultimately determined by surface morphology, it is technologically important to understand the mechanisms controlling morphology.
From an atomistic perspective, the surface morphology of a growing film is controlled by surface kinetic processes: dissociation, chemisorption, desorption, diffusion, step-edge attachment, and 2D nucleation, are the most basic ones; necessarily, some of these processes must occur before an atom or molcculc from the vapor is incorporated at the growth front. Under the normal supersaturation conditions which exist for MBE growth, surface kinetics is significantly limited, causing growth to occur far from a $2 \mathrm{D}$ quasi-equilibrium state. Consequently, even in the simplest of material systems, homoepitaxy, an understanding of the dynamical cvolution of the surface morphology is challenging.

Reflection high-energy electron diffraction (RHEED) has become the standard tool for characterization of films during MBE growth [2]. Models addressing the epitaxial growth of GaAs naturally attempt to compare with experiment by determining an oscillating quantity. This issue is broader than simply determining what RHEED measures. RHEED oscillations reveal a general pattern or characteristic in the growth behavior. Consequently, correlating the diffraction signa- 
ture with the surface morphology quite generally advances an understanding of epitaxial growth. Efforts aimed at modeling non-equilibrium film growth phenomena are particularly hindered by the lack of a real-space picture of the growth front as it evolves.

The goal of these experimental studies is to obtain a real-space picture of the evolution of
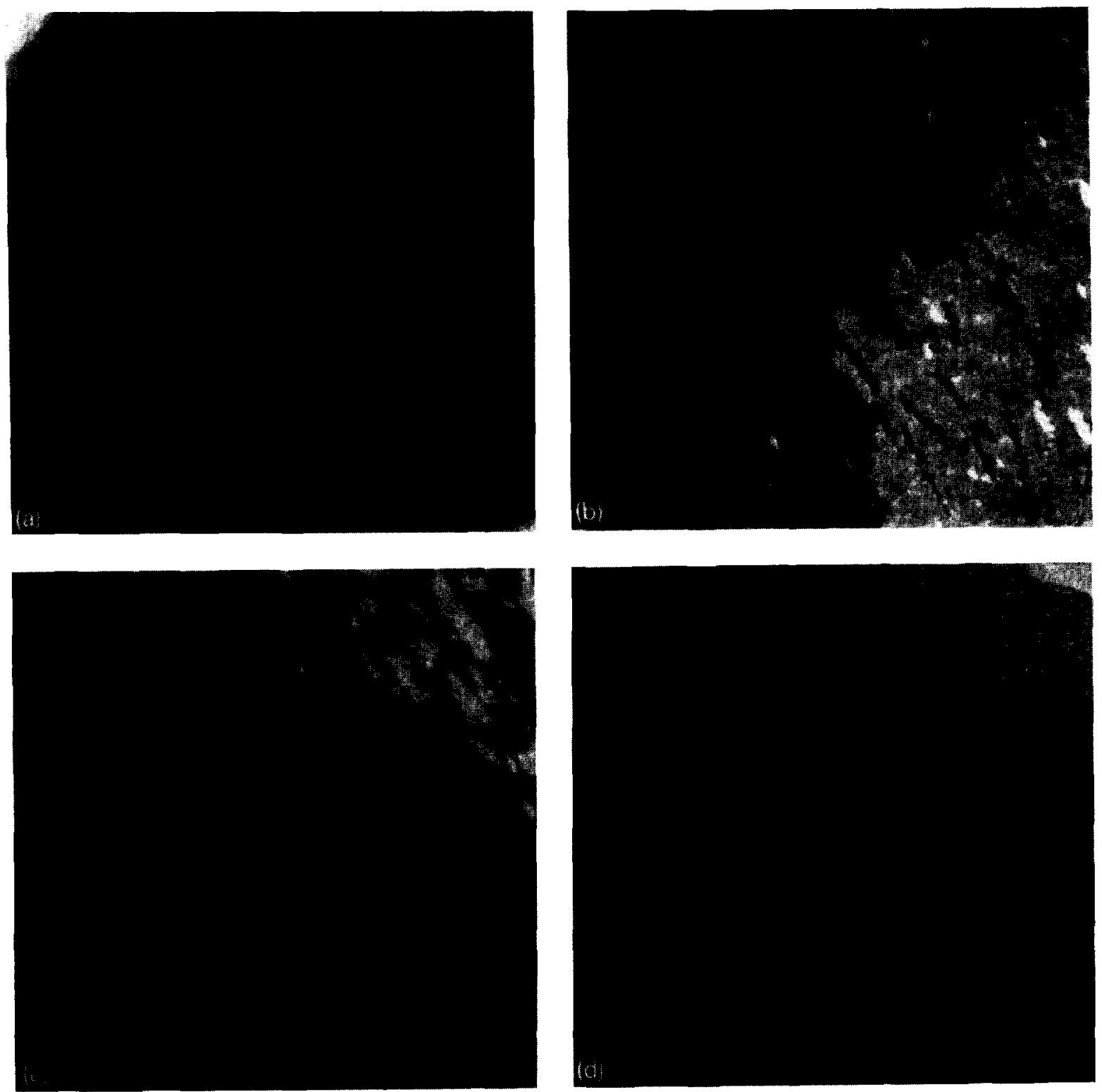

Fig. 1. (a) STM image of a GaAs(001) buffer layer. The scan range is $200 \times 200 \mathrm{~nm}^{2}$. The tunneling voltage $\left(V_{t}\right)$ was $+2.8 \mathrm{~V}$, applied to the sample and the tunneling current $\left(I_{\mathrm{t}}\right)$ was $80 \mathrm{pA}$. (b) STM image of GaAs(001) surface after termination of growth at the fourth RHEED maximum. (c) STM image of GaAs(001) surface after termination of growth at the fourth RHEED minimum. (d) STM image of GaAs(001) after $60 \mathrm{ML}$ have been deposited. Note that the local direction of the viscinality in (d) differs from that shown in the previous images. 
GaAs surface during growth and investigate its relationship to RHEED oscillations. In the posttransient regime where RHEED oscillations are no longer present, the surface evolution is also studied. In the interest of space, we present STM images of $200 \times 200 \mathrm{~nm}^{2}$ size. For a more comprehensive view of the surfaces, the readers are referred to large-scale STM images published elsewhere [3]. The experiment consists of initiating growth from a recovered surface and then terminating deposition at a specific point during the growth process. This procedure is then repeated for various termination points. Because the samples are removed from the STM for regrowth, no direct comparison can be made between any specific feature in the progression of the images. We have imaged large areas at multiple sites on multiple samples; the images shown are representative of the surface.

Deposition was performed in a standard ultra-high vacuum system, base pressure $7 \times 10^{-11}$ Torr. Effusion cells were used to produce both the $\mathrm{Ga}$ and $\mathrm{As}_{4}$ fluxes. Commercial GaAs(001) substrates were first chemically cleaned then loaded in the vacuum system where the oxide was removed at $580^{\circ} \mathrm{C}$ under an $\mathrm{As}_{4}$ flux. Prior to the experiments a $300 \mathrm{~nm}$ thick buffer layer was grown. The substrate temperature during deposition was $555^{\circ} \mathrm{C}$. The As-to-Ga pressure ratio was 15 and the deposition rate was $0.15 \mathrm{ML} / \mathrm{s}$. The sample miscut as determined by STM was approximately $0.15^{\circ}$. The direction and magnitude of the local vicinality was found to vary appreciably. The incident angle of the RHEED beam was approximately $0.9^{\circ}$ and corresponded closely to the "in-phase" Bragg condition. This diffraction condition was chosen to yield a reduction in specularly scattered electron intensity with the initiation of growth. The azimuthal angle corresponded to a beam direction parallel to [110].

A central technical point of the experiment was the ability to quench the surface morphology as it appeared during growth. To accomplish this, a resistively heated low thermal mass sample was used. The sample temperature could be reduced from that during growth $\left(555^{\circ} \mathrm{C}\right)$ to below $450^{\circ} \mathrm{C}$ in $1.5 \mathrm{~s} . \mathrm{A} \mathrm{LN}_{2}$-cooled baffle with a cooled shutter shrouds the sample [4]. The quench procedure has been described in a previous publication [5]. Upon the completion of quench, the sample was transferred in situ to the analysis chamber equipped with an STM. The RHEED intensity was recorded up to the time the transfer occurs. We found that the RHEED intensity is quite steady during this period. Holding the sample at $400^{\circ} \mathrm{C}$ appeared to eliminate physisorption of As while not allowing significant step-cdge motion [6].

Fig. 1a shows an STM image of a $200 \times 200$ $\mathrm{nm}^{2}$ area of a recovered surface. The terrace size is large and the step edges smooth. This is the GaAs surface as it appears before growth. Figs. $1 \mathrm{~b}$ and $1 \mathrm{c}$ are, respectively, images of the surface as it appcarcd at the fourth RHEED intensity maximum and the fourth RHEED intensity minimum during the oscillatory regime.

There is clearly a morphological change in the sample surface profile as it evolves from a RHEED maximum to a RHEED minimum. The surface quenched at an intensity maximum shows few islands on terraces and an approximatcly equal number of monolayer-deep holes. In contrast, the surface quenched at a RHEED minimum shows many two-dimensional islands on the terraces. There is a much lower density of monolayer-deep holes. The terrace edges for both samples are relatively smooth.

In order to correlate the morphological evolution we observe with the time variation of the RHEED specular intensity one must consider various proposed models for the interpretation of the diffraction process. One simple approach uses a kinematical approximation to determine the interaction of the scattered electrons with the surface [7]. In this picture the measured intensity is due to the interference of the electrons scattered from different terraces on the surface. At the correct incident angle (off-Bragg condition) this leads to an oscillation of the specularly reflected intensity due to a changing terrace occupation during deposition. As each growing layer proceeds from zero coverage through half filling and finally to a complete layer the specular intensity cycles through one period. A second, largely phenomenological, model which attempts to incorporate diffuse scattering has been proposed to 
explain RHEED oscillations. In this model the relevant quantity is not the terrace occupation but the step-edge length per unit area, termed step density [8]. Steps provide a mechanism for diffuse scattering of the electron beam. With an increase in the step density the specularly reflected intensity would decrease. As in the previous model, if the surface morphology cycles from islanded to flat then the RHEED intensity would vary accordingly. Monte Carlo growth simulations have shown an excellent correspondence between the step density and experimental RHEED data taken on vicinal surfaces [9]. The third explanation for RHEED oscillations during GaAs growth involves the dissociation dynamics of $\mathrm{As}_{2}$ of the surface [10]. By postulating a configuration-dependent reaction rate it is found that the $\mathrm{As}_{2}$ dissociation displayed an oscillatory behavior with the correct monolayer period. This model does not require that there be any differences in morphological step distribution during growth to account for the intensity oscillations.

There has been criticism of these models due to the incomplete treatment of dynamical scattering. It has been experimentally demonstrated that the behavior of the scattered electrons is a complicated function of both azimuthal and polar angles [11]. These data cannot be explained within a kinematical framework. In recent years some progress has been made towards a fully dynamical approach [12]. However, the surfaces so treated have been highly idealized and the connection with real systems remains tenuous.

Our STM data can be interpreted within the context of the step density model. The diffraction conditions correspond to the "in-phase" Bragg condition where electrons reflected from adjacent terraces constructively interfere. The specular intensity varies not because of interference but because of diffuse scattering from step edges [13]. There is a correspondence between films with higher step density and lower RHEED specular intensity at this diffraction condition. On closer inspection of the data it is natural to ask if the scattering from islands and holes should be weighted equally. Geometrically, the scattering from a small hole might be quite different than that from an island or terrace edge. We have calculated the specular scattering from holes and islands for the Bragg condition within a modified Born approximation [14]. It is found that holes less than $5 \mathrm{~nm}$ in diameter contribute far less to the diffuse scattering than do islands and terraces edges. As a first approximation, if the step density contribution from small holes is subtracted from the total then the agreement between this modified step density and RHEED intensity is quite strong, see fig. 2. After decay of the step density oscillations there continues to be a monotonic decrease in the RHEED intensity. This corresponds to the monotonic increase in step density seen in fig. 2 between 60 and $120 \mathrm{ML}$ deposition. Although the exact functional dependence of the intensity on step density is probably complicated, the physical mechanism of diffuse scattering from steps is clearly indicated.

To further investigate the growth process, we have also examined the sample surface after the decay of RHEED oscillations. Fig. 1d shows the surface after $60 \mathrm{ML}$ have been deposited and the RHEED oscillation amplitude has decayed to less than $5 \%$ of its original value. The sample is quenched in the same manner as before. The typical feature size has increased and in contrast to the earlier data the two-dimensional islands and terrace edges are now quite ramified. Fig. 3a displays the surface after deposition of $120 \mathrm{ML}$. The surface in fig. 1d has coarsened so much that it starts to roughen and nucleate $2 \mathrm{D}$ islands on

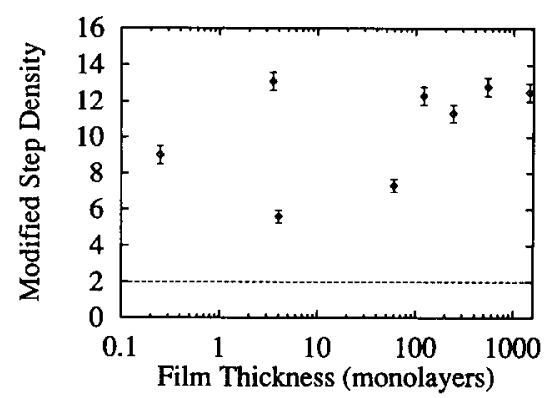

Fig. 2. Plot of the surface step densities (in units of $10^{-2}$ $\mathrm{nm}^{-1}$ ) as a function of thickness (in ML). The modified step density excludes the contribution from small holes less than 5 $\mathrm{nm}$ in diameter. The dashed line represents the starting growth substrate. 
top of the terraces. This may explain the increase in step density between $60-\mathrm{ML}$ deposited surface and that of 120 monolayers. Nevertheless, the overall morphology remains flat, with about four layers present. Surprisingly, further growth does not seem to increase the surface roughness, as evidenced by figs. $3 b-3 d$. These STM images obtained after depositing 240, 540 and 1450 monolayers (figs. $3 \mathrm{~b}, 3 \mathrm{c}$ and $3 \mathrm{~d}$, respectively) show essentially identical topography to that of the 120
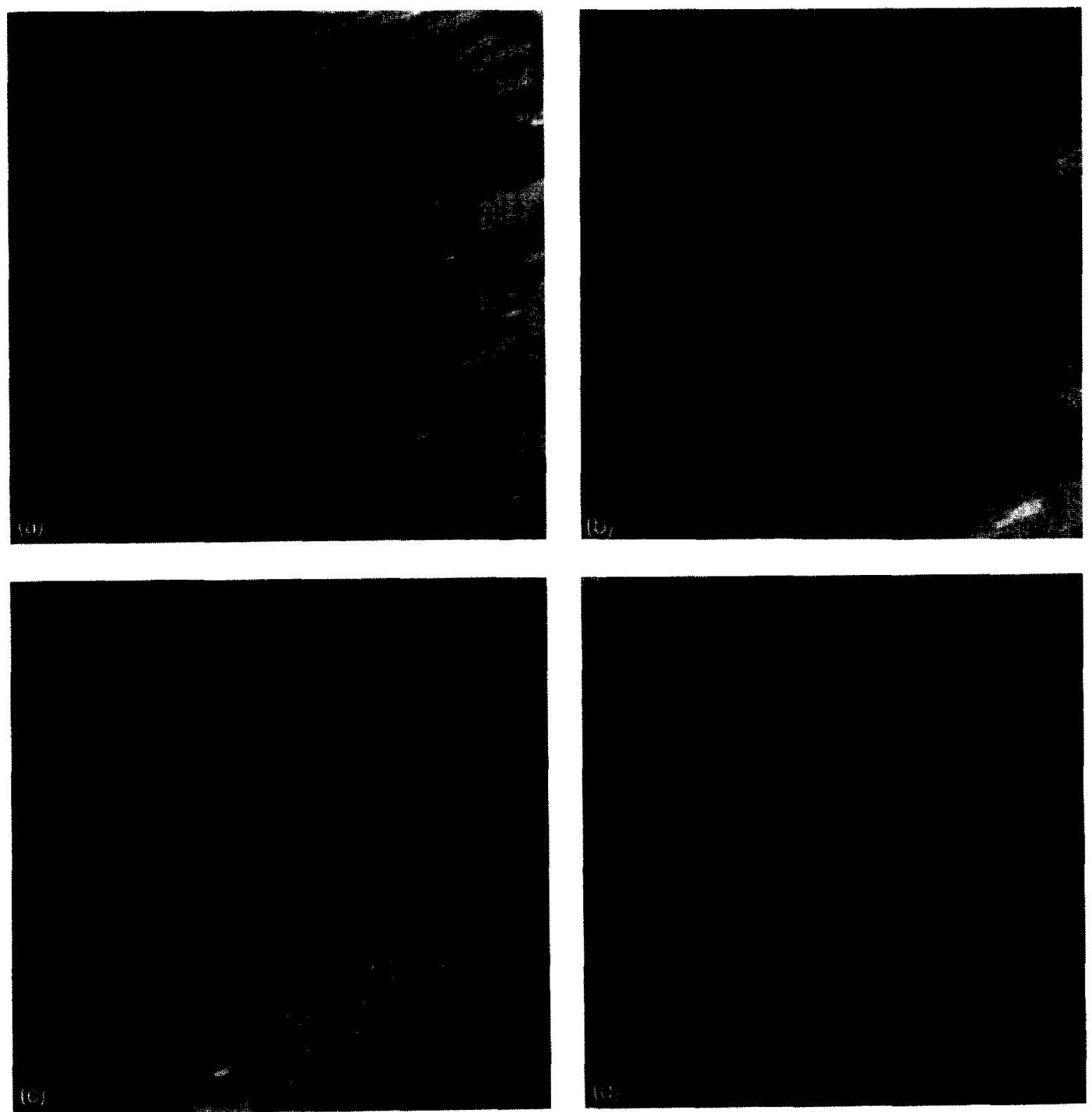

Fig. 3. (a) STM image of GaAs(001) after termination of growth of $120 \mathrm{ML}$. (b)-(d) STM images of GaAs(001) after deposition of 240,540 , and $1450 \mathrm{ML}$, respectively. The size of all images is $200 \times 200 \mathrm{~nm}^{2}$. Again, notice that the local vicinality varies slightly from one image to another. 


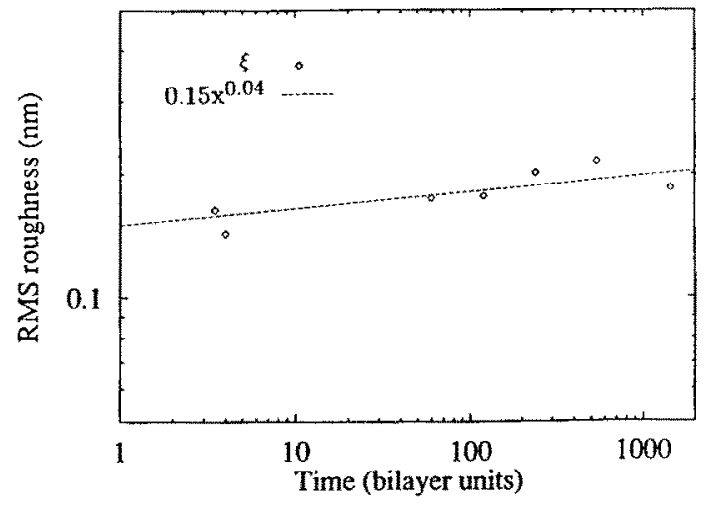

Fig. 4. RMS roughness (in $\mathrm{nm}$ ) of the surfaces as a function of deposition time (monolayer units). The time to deposit one monolayer is $6 \mathrm{~s}$. The rms roughness is defined as $\sqrt{(1 / N) \sum_{i}\left(h_{i}-\bar{h}\right)^{2}}$, where $h_{i}$ is the height and the sum is over a $200 \times 200 \mathrm{~nm}^{2}$ area.

ML film. The rms roughness of these surfaces is $\sim 2.5 \AA$. This points to a central feature of the data, that is the decay of the oscillation amplitude has occurred without an increase in the interface roughness. As shown in fig. 4, the interface width of the grown surface increases extremely slowly. Furthermore, the surface has achieved a steady-state configuration, through a balance of island nucleation and step flow, which evolves with a constant step density. In short, the surface has reached the step flow growth mode.

The question becomes: What is physically occurring on the surface when step flow is achieved? During growth there are three scenarios for the surface trajectory of an adatom [15]. It can either diffuse to an existing upward step or kink on a terrace and be incorporated, diffuse to an existing downward step and be incorporated in the lower terrace, or collide with another adatom and form a new stable island. If the formation of new stable 2D nuclei is dominant then the growth is called layer-by-layer [16]; however, if diffusion to an existing step dominates then the growth is termed step flow. The relative probability of an adatom to follow one of these trajectories is a complicated function of the substrate temperature, growth rate, and surface morphology. The experimental results indicate that evolution of growth of $\mathrm{Ga} \Lambda$ s can be characterized by a change in the relative probability to nucleate a new island versus attaching to an existing step edge. This evolution may be thought of as a dynamical transition to step-flow growth. What is remarkable is that even though the substrate temperature and growth rate are constant, the surface morphology has been transformed to allow a new growth mode.

However, this type of growth is not what is conventionally called step flow, i.e., a uniform progression of the steps across the surface. This classic step flow occurs when the substrate has a sufficient density of steps due to large miscut, and the adatoms have a large enough mobility. The new feature presented here is that deposition has dynamically generated a sufficient step density to allow for the change from layer-by-layer growth to step-flow growth mode. With this picture in mind one can extend the analysis to the study of kinetic roughening during film deposition. Results are presented clscwhcre [17].

In summary, we have studied the evolution of the GaAs surface during MBE growth. Starting from a recovered substrate, upon the initiation of growth the surface progresses through a transient regime, where cyclic changes in the step density are found, to a local steady state. We have shown a clear connection between the surface morphology and RHEED oscillations. Specifically, with in-phase diffraction conditions, chosen to eliminate the kinematic contribution, the RHEED intensity oscillations are shown to correlate well with the surface step density. A striking result is that the decay of the RHEED oscillations is not duc to an increase in surface width, but the dynamical evolution of the surface to step-flow growth, defined as a local steady state with a constant step density.

The authors would like to acknowledge M.B. Elowitz for his assistance. We are grateful to RHK technology and Burleigh Instruments for equipment grants. This work has been supported by the following grants: N00014-89-J-1519, NSF/DMR-8857828, and the Sloan Foundation. 


\section{References}

[1] A.Y. Cho, Thin Solid Films 100 (1983) 291, and references therein.

[2] General review of RHEED techniques can be found in: Reflection High Energy Electron Diffraction and Reflection Electron Imaging of Surfaces, NATO ASI Series B 188, Eds. P.K. Larsen and P.J. Dobson (Plenum, New York, 1988)

[3] J. Sudijono, M.D. Johnson, M.B. Elowitz, C.W. Snyder and B.G. Orr, Surf. Sci. 280 (1993) 247.

[4] B.G. Orr, C.W. Snyder and M.D. Johnson, Rev. Sci. Instrum. 6 (1991) 1400.

[5] J. Sudijono, M.D. Johnson, C.W. Snyder, M.B. Elowitz and B.G. Orr, Phys. Rev. Lett. 69 (1992) 2811.

[6] J. Singh and K.K. Bajaj, J. Vac. Sci. Technol. B 2 (1984) 576. The smallest feature observed in our data is about two-thirds of a unit cell.

[7] P.R. Pukite, C.S. Lent and P.I. Cohen, Surf. Sci. 161 (1985) 39.
[8] S. Clarke and D.D. Vvedensky, Phys. Rev. Lett. 58 (1987) 2235.

[9] T. Shitara, D.D. Vvedensky, M.R. Wilby, J. Zhang, J.H. Neave and B.A. Joyce, Appl. Phys. Lett. 60 (1992) 1504.

[10] S.V. Ghaisas and A. Madhukar, Phys. Rev. Lett. 56 (1986) 1066.

[11] P.J. Dobson, B.A. Joyce, J.H. Neave and J. Zhang, J. Cryst. Growth 81 (1987) 1.

[12] T. Kawamura and P.A. Maksym, Surf. Sci. 161 (1985) 12.

[13] T. Shitara, Thesis, Imperial College, 1992.

[14] K.T. Leung, J. Sudijono, M.D. Johnson, C.W. Snyder, A.W. Hunt and B.G. Orr, to be published.

[15] The substrate temperature is sufficiently low such that the probability of diffusion from a highly coordinated site such as a terrace edge is extremely low.

[16] J.H. Neave, B.A. Joyce, P.J. Dobson and N. Norton, Appl. Phys. A 31 (1983) 1.

[17] M.D. Johnson, C. Orme, A.W. Hunt, D. Graff, J. Sudijono, L.M. Sander and B.G. Orr, preprint. 